\title{
MAGNETOELASTIC EFFECTS AND MAGNETIC ANISOTROPY IN SUPERLATTICE SYSTEM
}

\author{
R. JAROSZEWICZ AND Z. BĄK \\ Institute of Physics, Pedagogical University \\ Armii Krajowej 13/15, 42-201 Częstochowa, Poland
}

\begin{abstract}
In this contribution, we will discuss the effect of interface misfit strain on magnetic coupling of ferromagnetic layers, across intervening, metallic spacer in the epitaxial superlattice system. We calculate the interface lateral lattice deformation, due to ionic radii misfit within the Frenkel-Kontorova model and derive its influence on surface magnetic anisotropy and electronic properties of the nonmagnetic spacer. We will study also the origin of the interface magnetoelastic asymmetry in superlattices and its effect on magnetic coupling between magnetic layers. Also the magnetoelastic contribution to the in-plane exchange coupling is widely discussed.
\end{abstract}

PACS numbers: 75.70.Cn, 75.70.Ak

Using modern manipulation opportunities, the magnetic superlattices (MSLs) may be fabricated to have properties which might not be otherwise attainable. Understanding the physical mechanisms that govern magnetic behaviour of MSL is thus crucial for engineering desired properties of electronic devices. At present, the main theoretical interest is focused on the description of the long period, RKKY-reminiscent interlayer coupling and giant magnetoresistance [1,2]. Almost all theoretical studies of the problem are based on restrictive assumption on the system, the layers are supposed to be coherent, i.e. it is assumed to be uniform in the in-plane directions. This is true for those heteroepitaxial MSLs, for which the elemental constituents exhibit a negligible ionic radii mismatch. When the ionic radii differ by a few percent, additional tensile arises in the interface region or compressive strains relaxed through the misfit dislocation. For a higher value of the ionic radii mismatch (e.g. in the $\mathrm{Co} / \mathrm{Au}$ system equals about $14 \%$ [3]), the single crystalline superlattice can be obtained only for samples with a Co layer thickness below about $10 \AA$. At higher ionic radii misfit the interface biaxial tension is so large that the heteroepitaxial growth cannot occur. The aim of the paper is to study the elastic and magnetoelastic effects within the interface regions. In the following we will consider the influence of interface strains on the surface magnetic anisotropy and the magnetic coupling in the MSL systems.

The basal-plane lattice mismatch between A and B metallic constituents produces lateral tensile/compressive strains and as the result of that there is an 
in-plane lattice distortion associated with these strains [4]. The influence of the lateral strains on the electronic structure can be calculated under assumption that the local distortions are described by the Frenkel-Kontorova model. The Frenkel-Kontorova model consists of a chain of atoms connected by a spring situated in a sinusoidal potential well and has a potential energy [5]

$$
V=\frac{1}{2} \alpha \sum_{n}\left(x_{n+1}-x_{n}-b\right)^{2}-V_{0} \sum_{n} \cos \left(2 \pi x_{n} / a\right)
$$

where $\alpha$ is the force constant of a spring, $V_{0}$ is the strength of the sinusoidal potential well, $b$ and $a$ are the natural periods of the chain and the sinusoidal potential well, respectively, and $x_{n}$ is the position of the $n$-th atom. The equation of motion for lattice phonons reads,

$$
m \ddot{U}_{n}=-\alpha\left(2 U_{n}-U_{n+1}-U_{n-1}\right)-V_{0}(2 \pi / a)^{2} \cos \left(2 \pi \bar{x}_{n} / a\right) U_{n}
$$

where $\mathrm{U}_{n}$ are the displacements from the equilibrium positions $x_{n}$ satisfying periodic boundary conditions. Solving Eq. (2) within the perturbation theory in the first order approximation we obtain

$$
\bar{x}_{n} \approx x_{1}^{0}+(n-1) b-\gamma \sin (2 \pi / a)\left[x_{1}^{0}+(n-1) b\right],
$$

where $x_{1}^{0}$ is the zeroth-order position of the chain and

$$
\gamma=V_{0}(2 \pi / a)\left[4 \alpha \sin ^{2}(\pi b / a)\right]^{-1}
$$

In the case of lattice mismatched heteroepitaxy, an initial uniform biaxial coherency strain in the magnetic film is eventually relieved above a critical thickness $t_{c}$ by the nucleation of misfit dislocations [6]. For $t>s$ (where $s$ is the misfit dislocations spacing) the film experiences an average uniform equilibrium strain. However, for $t<s$ the nonuniform character of the strain field cannot be neglected. In view of Eq. (3) one would expect a sinusoidal modulation of the in-layer lattice distortion and a similar variation of the in-plane stress. This inhomogeneous distortion affects both the magnetic interaction $[7,8]$ and the magnetic anisotropy.

Let us study the effect of nonuniform strain on the magnetic surface anisotropy in the SL system when the nonuniform interface ion distortions are given by Eq. (3). We assume the Néel-type surface anisotropy dominates in the system under consideration [9]. Néel proposed that the ions in the reduced symmetry positions will give rise to anisotropies, which are absent in the bulk systems. In the Néel model, the magnetic pair-interaction energy between ions is expanded in Legendre polynomials. In the vicinity of the interface we can write [9]

$$
E(r, \psi)=G(r)+L(r)\left(\cos ^{2} \psi-\frac{1}{3}\right)+Q(r)\left(\cos ^{4} \psi-\frac{6}{7} \cos ^{2} \psi-\frac{3}{35}\right),
$$

where $\psi$ is the angle between the bond axis and the magnetization at the interface $\boldsymbol{M}_{\mathrm{s}}$, while $\boldsymbol{r}$ denotes the distance between pair of atoms. The strain dependence of the anisotropy (5) is described within the Néel model by taking into account the strain induced changes in $r$ and $\psi$. The coefficients of Eq. (5) are functions of the distance $r$ between pairs of the magnetic ions and can be expanded in power series of bond strain $\epsilon$

$$
L_{i}(r)=L_{i}\left(r_{0}\right)+\left(\frac{\mathrm{d} L_{i}}{\mathrm{~d} r}\right) \epsilon r_{0}+\ldots
$$


where $L_{1,2}$ denotes interaction between first-nearest and second-nearest neighbours, respectively. For given substrates, the values of $L_{i}\left(r_{0}\right)$ can be related to the magnetic anisotropy constant $K_{1}$ and the magnetoelastic coefficients $B_{1}$ and $B_{2}$ of the bulk material [9].

$$
\Delta_{\text {surf }}^{(001)}=-\frac{8}{3}\left(\frac{\mathrm{d} L_{i}}{\mathrm{~d} r}\right) r_{0} \epsilon \cos ^{2} \theta
$$

with $\theta$ being the angle between normal to the interface and the direction of effective magnetization. If the local strain $\epsilon$ is associated with the nonuniform surface lattice distortion by the oscillatory part of the Frenkel-Kontorova solution (3) then the surface anisotropy also oscillates in space between negative and positive values. The surface anisotropy superimposes with the bulk contribution and in the vicinity of the interface we can write.

$$
E_{\text {anis }}=E_{\text {anis }}^{\text {bulk }}+\frac{1}{t} \Delta E_{\text {anis }}^{(001)} \text {. }
$$

Provided that the surface anisotropy dominates the bulk contribution, the magnetization easy axis varies periodically between two perpendicular directions. As it has been shown [9] for the bcc lattice, the strained bulk anisotropy has the same sign and its magnitude is twice that of the surface contribution given by Eq. (7). As a result of that there appears 2D modulation of the magnetic anisotropy which generates a domain structure. Within one domain the surface anisotropy favours magnetization being either parallel or perpendicular to the growth direction.

From the solutions of the Frenkel-Kontorova model one can draw conclusion that in some cases the strains at two interfaces of a given layer are not symmetrical. Having the solutions (1-4), let us consider strains at both interfaces of a layer in a superlattice composed of $M e_{1}$ and $M e_{2}$ constituents. A typical metallic superlattice is a system of multiple repeated $M e_{1} / M e_{2}$ and $M e_{2} / M e_{1}$ interfaces. In the first case the $M e_{1}$ component serves as the substrate and the $M e_{2}$ is the overlayer, while for the other case the role interchanges. This means that in one case the interface described within the Frenkel-Kontorova model with potential (1) is determined by the parameters $V_{0}^{1}$ and $\alpha_{2}$, while for the other case by $V_{0}^{2}$ and $\alpha_{1}$. In view of the result (4) one would expect that for the relatively thick layers (i.e. when interface strains relax across the spacer according to the formula (8)) there should be a strain asymmetry between $M e_{1} / M e_{2}$ and $M e_{2} / M e_{1}$ interfaces. Really, for the case of $\mathrm{Au} / \mathrm{Co}$ and $\mathrm{Au} / \mathrm{Ag}$ superlattices such an asymmetry has been observed [10].

In summary, since other kinds of stress are usually of minor importance we have focused on the interface stress resulting from the lattice mismatching of the adjacent layers in the superlattice system and calculated magnetic anisotropy due to this strain. For simplicity we have neglected the contribution of roughness at interfaces whose influence on the magnetic anisotropy is always important. We have proved that for the bcc lattice there can arise an interesting situation, when the magnetic anisotropy oscillates between the in-plane and perpendicular to plane directions. Thus, the magnetic anisotropy that arises due to the interface strain should induce a periodic array of magnetic domains, with the domain magnetization perpendicular to each other. The detailed analysis of the Frenkel-Kontorova 
solutions suggests that there can be a strain asymmetry at both interfaces of a given layer within the superlattice system.

\section{Acknowledgments}

A financial support from the Committee for Scientific Research is gratefully acknowledged.

\section{References}

[1] P. Bruno, Phys. Rev. B 52, 411 (1995).

[2] W. Gruhn, Z. Bak, R. Jaroszewicz, Acta Phys. Pol. A 89, 417 (1996).

[3] L. Wu, K. Shintaku, T. Shinjo, N. Nakayama, J. Phys. Condens. Matter 5, 6515 (1993).

[4] R. Żuberek, H. Szymczak, R. Krishnan, M. Tessier, J. Phys. (France) C 8-49, 1761 $(1988)$.

[5] J.B. Sokolov, J.E. Sacco, J.F. Weisz, Phys. Rev. Lett. 41, 1561 (1978).

[6] R.C. O'Handley, S.-W. Sun, J. Magn. Magn. Mater. 104-107, 1717 (1992).

[7] Z. Bąk, W. Gruhn, R. Jaroszewicz, J. Magn. Magn. Mater. 148, L387 (1995).

[8] R. Jaroszewicz, Z. Bąk, W. Gruhn, Acta Phys. Pol. A 89, 421 (1996).

[9] D.S. Chuang, C.A. Ballentine, R.C. O'Handley, Phys. Rev. B 49, 15084 (1994).

[10] T. Kinegatsu, K. Sakai, Phys. Rev B 48, 4140 (1993). 